\title{
Improved MDA, a case for de-garbling SSR mode $\mathrm{S}$ replies
}

\author{
N. Petrochilos \\ CRESTIC, University of Reims, \\ BP 1039, 51687 REIMS Cedex2, France \\ nicolas.petrochilos@gmail.com
}

\author{
E.G. Piracci, G. Galati \\ D.I.E. and Vito Volterra Centre \\ Tor Vergata University, Via del Politecnico, 1 Rome, Italy \\ galati@uniroma2.it, piracci@ing.uniroma2.it
}

\begin{abstract}
Multilateration (MLAT) and Automatic Dependent Surveillance - Broadcast (ADS-B) systems exploiting the Secondary Surveillance Radar (SSR) channel suffer from garbling. This means that if two or more mode $S$ signals impinge on the receiver at the same or very near time they could not be decoded. To alleviate this problem, many solutions have been proposed, one in particular [1] is effective for a large variety of scenarios, but excluding the cases when the replies are too much separated in time. Recently a paper [2] focused on linear algebra presented a potential solution for this case. In this work we present a practical application for the case of two mode $S$ signals.
\end{abstract}

Keywords- Mode S, SSR, ADS-B, Multilateration, signals separation, algebraic algorithm, joint diagonalisation

\section{INTRODUCTION}

Following the legacy from the military IFF (Identification Friend or Foe) systems and then by the secondary surveillance radar (SSR) [3], today the $1090 \mathrm{MHz}$ channel is widely used for air traffic (but also in airport for vehicular traffic) surveillance. Nowadays, the $1090 \mathrm{MHz}$ signals are also used in systems such as ADS-B, Multilateration (MLAT), and likely to be integrated by the TIS-B stations, which provide information on non-ADS-B aircraft. For these reasons the integrity and the efficiency of these systems have become more and more important.

In a typical high-density airspace, an increasing number of transponders (airborne or vehicular) transmit signals at $1090 \mathrm{MHz}$, either as replies to the SSR stations (conventional and Mode S), or spontaneously ('Squitter'). This leads to the case where several Mode S replies can impinge on the receiver at the same time and be corrupted or "garbled". Historically, most of these receiving systems had one antenna, with sum and difference beams; some of the more recent systems for MLAT and ADS-B only have a nondirectional antenna [4]. Having an antenna array, on the other side, opens the possibility to use source separation methods, as in $[1,5,6,7]$. Other groups have also worked on this approach $[2,8,9]$, and a good survey can be found in [10].

The promising Manchester Decoding Algorithm (MDA) [1], suffers from one critical weakness: the failure ratio raises when the overlap between replies decreases. In order to overcome this problem, we derived at Tor Vergata University a strategy which consists of using a detector to evaluate the number of sources as a function of time, and depending the time delay to go on either with the MDA or with the Projection Algorithm (PA, [6]), which is inefficient for totally overlapping replies. In [2], the authors presents a solution, Subspace-Intersection MDA (SI+MDA), for a more complicated problem: considering a much longer interval of time, up to 200 microseconds, and 5 short mode $\mathrm{S}$, all overlapping three by three at most, to separate all the replies. The basis of the separation is the MDA, but in SIMDA there are two improvements, a pre-processing step, and a linear algebra step, which permits to decimate the length by three and to push out some replies. The authors in [2] are mainly interested in the linear algebra step, which is unfortunate since this step foreshadow the actual benefit of the pre-processing step. Moreover their scenario needs a minimum of 5 replies in the 200 microseconds window. We derived the probability to have $n=\{2, \ldots, 5\}$ replies in 200 microseconds (see Figure 1) as a function of rate of number of messages per seconds, named FRUIT.

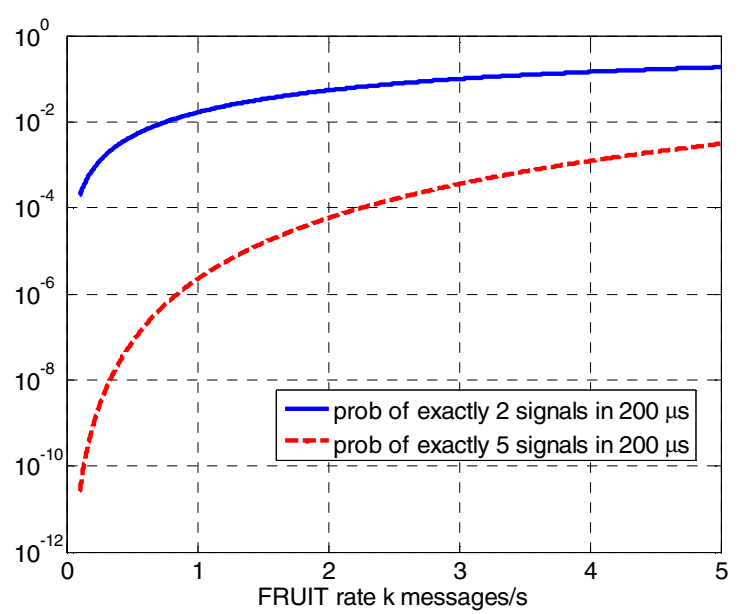

Figure 1. - Probability of 2 and 5 signals in $200 \mu$ s as a function of FRUIT rate measured at Tor Vergata University. 
Given that the observed rate at Roma airports is between 500 and 1000 replies per seconds [11], the ratio between the two cases is 10.000 , and the 5-reply event occurs on average 5 times a day. Therefore the focus of this article is to propose an improved MDA, henceforth called the FoldMDA, which is a more practical and "simplified" version of the SI+MDA. Its main point is to detect the number of sources as a function of time. When a large time delay between two mode $\mathrm{S}$ replies is detected the idea is to fold the data, hence the name fold-MDA. Although the FoldMDA fits better operational uses in the Roma airports conditions, we agree that the SI+MDA could be useful in higher density airspace.

In the next section, we describe the data and present the Manchester encoding property, the MDA, and the FoldMDA. Next, we present some simulations that allow assessing the quality of the Fold-MDA as compared to the competitors. Finally, a case of study with a real signal is presented, assessing the application of MDA and of FoldMDA. The signal was recorded using a system, called TDR (Transponder Data Recorder) [12], complying with ICAO and RTCA requirements [13] [14], which has been designed and developed by the Radar and Navigation group, RadarLab, at Tor Vergata University, in collaboration with the Microwave Laboratory at the University of Calabria, Rende (CS), Italy.

\section{DATA MODEL, MANCHESTER ENCODING PROPERTY, AND MDA}

\section{A. Data model}

The precise model can be found in [1], but also in $[3,5,6,7,8,9,10]$. To be quick, let us admit that we receive $d$ independent source signals on an $m$ elements array antenna and. The base band signals are sampled at period $T$ and stacked in vectors $\mathbf{x}[\mathrm{n}]($ size $m$ ). After collecting $N$ samples, the observation model is:

$$
\mathbf{X}=\mathbf{M S}+\mathbf{N},
$$

where $\mathbf{X}=[\mathbf{x}[1], \ldots, \mathbf{x}[\mathrm{N}]]$ is the $m \times N$ received signal matrix, and the $d \times N$ matrix $\mathbf{S}=[\mathbf{s}[1], \ldots, \mathbf{S}[\mathrm{N}]]$ is the source matrix, with $\mathbf{s}[\mathrm{n}]=\left[\mathrm{s}_{1}[\mathrm{n}], \ldots, \mathrm{s}_{\mathrm{d}}[\mathrm{n}]\right]^{\mathrm{T}}$ being the stacking of the $d$ source signals, with zero-padding to account the limited time extend of the sources. $\mathbf{N}$ is the $m \times N$ noise matrix, whose elements are temporally and spatially white. $\mathbf{M}$ is the $m \times d$ mixing matrix that contains the array signatures and the complex gains of the sources. We assume that the replies are independent. Finally, we assume $d<m$ and $\mathbf{M}$ full column rank.

\section{B. Manchester encoding property}

A Mode S reply/squitter contains 56 (short) or 112 (long) binary symbols $b_{n}$. The symbol period is $1 \mu s$, and each symbol is made up by two $0.5 \mu s$ chips. Figure 2 shows a schematic view of the time intervals for the preamble and the data block, and also shows the concept of superimposed signals. The bit are Manchester encoded, that is the $b_{n}=0$ is coded as $\mathbf{b}_{n}=[0,1]$, and the $b_{n}=1$ is coded as $\mathbf{b}_{n}=[1,0]$.
A Mode S SSR reply/squitter is formed by a preamble $\mathbf{p}=$ $[1,0,1,0,0,0,0,1,0,1,0,0,0,0,0,0]$, followed by the encoded data: $\mathbf{b}=\left[\mathbf{p}, \mathbf{b}_{1}, \mathbf{b}_{2} \ldots \mathbf{b}_{56 / 112}\right]$, with a total length of 128 (short reply) or 240 (long reply) elements (64 or 120 $\mu s)$. The Mode S reply/squitter has the form:

$$
\mathrm{b}(t)=\sum_{n=0}^{127 / 239} \mathrm{~b}[n] p(t-n T)
$$

where $p(t)$ is a rectangular pulse of width $T=0.5 \mu \mathrm{s}$.

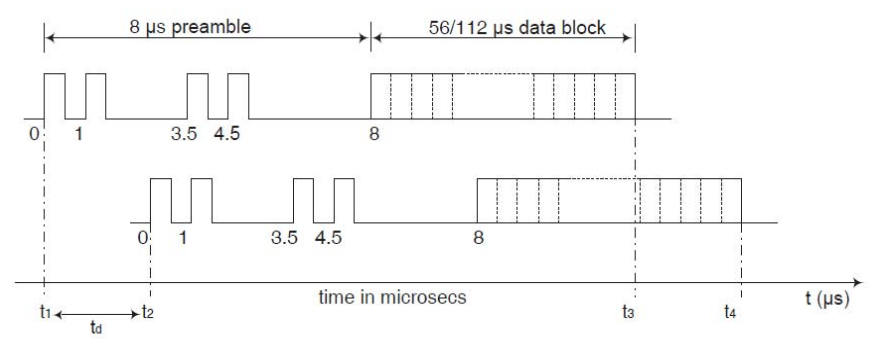

Figure 2. - Two Mode S replies with a time delay of $t_{d}=t_{2}-t_{I}$

It follows an interesting property: if the data are sampled at $T_{c}=0.5 \mu$ s, i.e. the length of a chip, or any of its multiples, the product of any three consecutive $T_{c}$-spaced samples is always equal to zero:

$$
b\left(t-T_{c}\right) \cdot b(t) \cdot b\left(t+T_{c}\right)=0
$$

And by extension for all mode $\mathrm{S}$ sources we have the following property:

$$
s_{i}\left(t-T_{c}\right) \cdot s_{i}(t) \cdot s_{i}\left(t+T_{c}\right)=0
$$

Using this property one can derive the MDA algorithm.

\section{Manchester Decoding Algorithm}

The MDA algorithm has been presented in [1], and aims at finding the $d$ beamformers $\mathbf{w}_{\mathbf{i}}$ such that for all $i$ in $\{1, d\}$, we have:

$$
\hat{\mathbf{s}}_{i}=\mathbf{w}_{\mathrm{i}}^{\mathrm{H}} \cdot \mathbf{x} \approx \mathbf{s}_{i}
$$

By properties (3) and using the adopted data model we obtain:

$$
[\mathbf{x}[\mathrm{n}+1] \otimes \mathbf{x}[\mathrm{n}] \otimes \mathbf{x}[\mathrm{n}-1]]^{H}(\mathbf{w} \otimes \mathbf{w} \otimes \mathbf{w})=\mathbf{0}
$$

Defining the matrix $\mathbf{P}$, of dimension $(N-2) \cdot d^{3}$, as the stack of rows $[\mathbf{x}[\mathrm{n}+1] \otimes \mathbf{x}[\mathrm{n}] \otimes \mathbf{x}[\mathrm{n}-1]]^{H}$ for $n=2, \ldots, N-1$, it follows that:

$$
\mathbf{P} \mathbf{w}^{\diamond}=0, \mathbf{w}^{\bullet}=\mathbf{w} \otimes \mathbf{w} \otimes \mathbf{w}
$$

For $d$ independent sources there are $d$ linearly independent separating beamformers $\mathbf{w}_{\mathbf{i}}$ and then $d$ linearly independent vectors $\mathbf{w}_{\boldsymbol{i}}^{\circ}$. By construction, vectors $\mathbf{w}_{\boldsymbol{i}}^{\circ}$, belong to the kernel of $\mathbf{P}$. The kernel of $\mathbf{P}$ is equal to the sub-space spanned by $\left\{\mathbf{w}_{\boldsymbol{i}}^{\diamond}, i=1 \ldots d\right\}$. By a SVD, a basis $\left\{\boldsymbol{y}_{\boldsymbol{i}}\right\}$ of $\mathbf{P}$ is estimated. It is composed by a linear combination of the 
vectors $\mathbf{w}_{\boldsymbol{i}}^{\circ}$. Thus, the fundamental step of the algorithm is to estimate the linear combination of $\left\{\mathbf{w}_{\boldsymbol{i}}^{\circ}, i=1 \ldots d\right\}$ that best fit $\left\{\boldsymbol{y}_{\boldsymbol{i}}\right\}$. This is a joint diagonalization problem in three dimensions. Finally, vectors $\mathbf{w}_{\mathbf{i}}$ are derived by the corresponding $\mathbf{w}_{\boldsymbol{i}}^{\bullet}$.

The algorithm steps can be resumed as:

1. Perform an SVD of the received data $\mathbf{X}$, to detect the number of source and prewhiten the data.

2. Construct the matrix $\mathbf{P}$.

3. Perform another SVD on $\mathbf{P}$, to extract its kernel, $\mathbf{U}$, which is of size $d$.

4. The joint diagonalization of the vectors of $\mathbf{U}$ gives the beamformers $\mathbf{w}_{\mathbf{i}}$.

The last step can use either the original method from [1], or the improved method presented in [15]. The MDA is quite robust, fast and efficient. It only suffers from a critical drawback which arises when the reply are weakly overlapping. In the original paper [1], there was a $1 \%$ failure at $30 \mu \mathrm{s}$ time delay, this failure rate is linear in log-scale and reaches $10 \%$ at $60 \mu$ s time delay, see figure 3 .

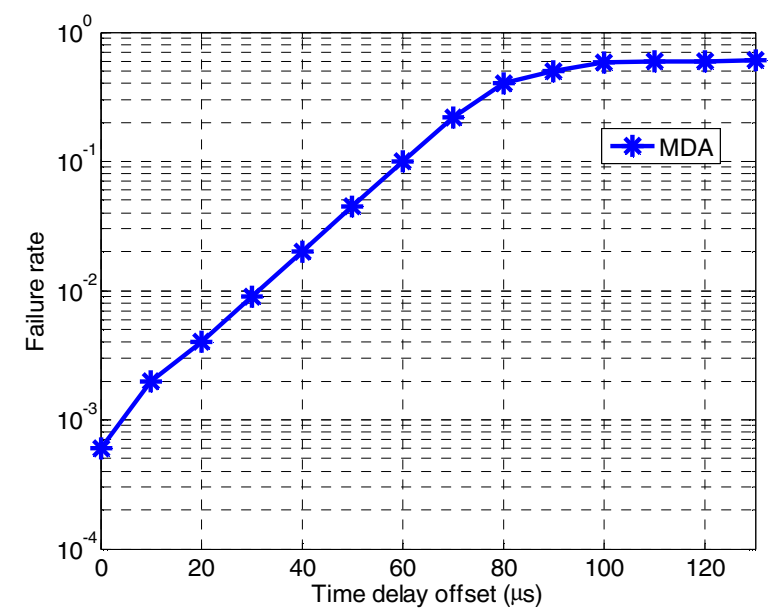

Figure 3. - MDA failure rate as a function of the time delay - simulations results (from [1])

This weakness originates from the step 3 of the algorithm, the fact that the matrix $\mathbf{P}$ is of rank $\left(d^{3}-d\right)$ and has a kernel of size $d$ is no longer valid when the replies do not overlap. In this case the kernel has a bigger size, and the estimation of the $d$ smallest eigenvalues is mistaken because of the noise. The interested reader can look for the proof in the appendix I from [1].

\section{Fold-MDA}

To overcome this problem, we propose the Fold-MDA. The concept is very simple: since the data does not overlap enough, we slice into two slots of length of $75 \mu \mathrm{s}$ for a mixture of short mode $\mathrm{S}$, and add these two slots into one. Since the slot are of length $75 \mu \mathrm{s}$, a given short mode $\mathrm{S}$ cannot overwrite itself.
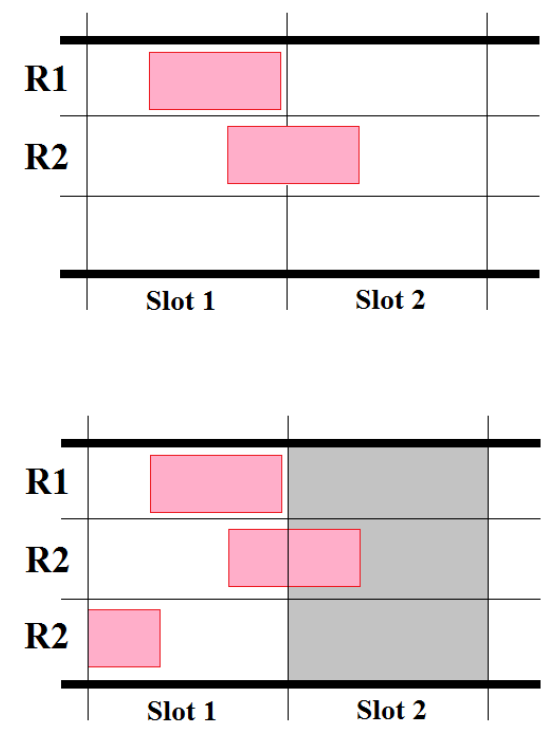

Figure 4. - Folding step

Figure 4 illustrate quite well the concept, where the first reply is complete over the first slot, whereas the second reply spans over two slots. Because the length of the slot is larger than the length of a mode S reply, we observe that the second reply is not rewritten.

Consider Equation (1), $\mathbf{X}=\mathbf{M} \mathbf{S}+\mathbf{N}$, that contains two slots, and denote $\mathbf{X}_{1}$ the matrix that contains the $\mathrm{N} / 2$ former samples, and $\mathbf{X}_{2}$ the latter N/2 samples. We define the Fold data matrix as:

$$
\mathbf{X}_{\mathrm{F}}=\mathbf{X}_{1}+\mathbf{X}_{2}
$$

Let us take the same notation on the signal matrix: $\mathbf{S}$ which gives then $\mathbf{S}_{\mathbf{1}}$ and $\mathbf{S}_{\mathbf{2}}$; and on the noise matrix. The equation becomes:

$$
\mathbf{X}_{\mathrm{F}}=\mathbf{M S}_{1}+\mathbf{M S}_{2}+\mathbf{N}_{1}+\mathbf{N}_{2}=\mathbf{M S}_{\mathrm{F}}+\mathbf{N}_{\mathrm{F}}
$$

In (8) $\mathbf{S}_{\mathbf{F}}$ is the sum of $\mathbf{S}_{\mathbf{1}}$ and $\mathbf{S}_{\mathbf{2}}$ (resp. $\mathbf{N}$ ). We note that the rank of $\mathbf{X}_{\mathbf{F}}$ is the same of $\mathbf{X}$, and that the beamformers of $\mathbf{X}$ will be the same for $\mathbf{X}_{\mathbf{F}}$. This is the basis of the algorithm, now the first and the second reply have a much larger overlap and the MDA would not fail. The algorithm follows as:

1. Construct matrix $\mathbf{X}_{\mathbf{F}}$

2. Apply MDA to $\mathbf{X}_{\mathbf{F}}$ and derive $\left\{\mathbf{w}_{\mathbf{1}}, \mathbf{w}_{\mathbf{2}}\right\}$

3. Apply the beamformers $\left\{\mathbf{w}_{\mathbf{1}}, \mathbf{w}_{\mathbf{2}}\right\}$ to $\mathbf{X}$

\section{SIMULATION RESULTS}

In order to evaluate the Fold-MDA, some simulations were done with a batch of data of 300 samples at a sampling rate $\mathrm{T}_{\mathrm{s}}=0.5 \mu \mathrm{s}$, so, duration of $150 \mu \mathrm{s}$, and slots of $75 \mu \mathrm{s}$. We used a four element array with an inter-distance of halfwavelength $(13 \mathrm{~cm})$ to simulate the reception of two sources.

The angles of arrival were $\{70,90\}$ degrees from the broadside, the residual frequency $\pm 50 \mathrm{kHz}$, the input $\mathrm{SNR}$, 
$10 \mathrm{~dB}$. The results were been evaluated in terms of success rate. A success is declared if it is possible to recover two independent sources after the algorithm application with an output SINR above $6 \mathrm{~dB}$.

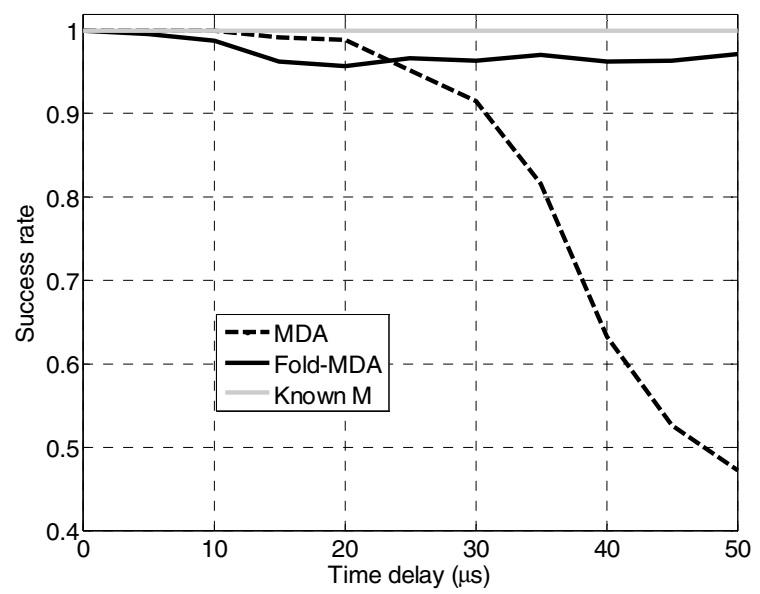

Figure 5. - Success rate as a function of time delay between the replies.

From Figure 5, where the time delay is varying, we can confirm that the Fold-MDA overcomes the limitation of MDA at small overlap (for time delays more than $30 \mu \mathrm{s}$ ). The solution with known $\mathbf{M}$ is always perfect.

In the next example, we fix the time delay at $30 \mu$ s, and we vary the input SNR from $2.5 \mathrm{~dB}$ to $20 \mathrm{~dB}$. The angles of arrival and the residual frequencies are the same as in the first simulation.

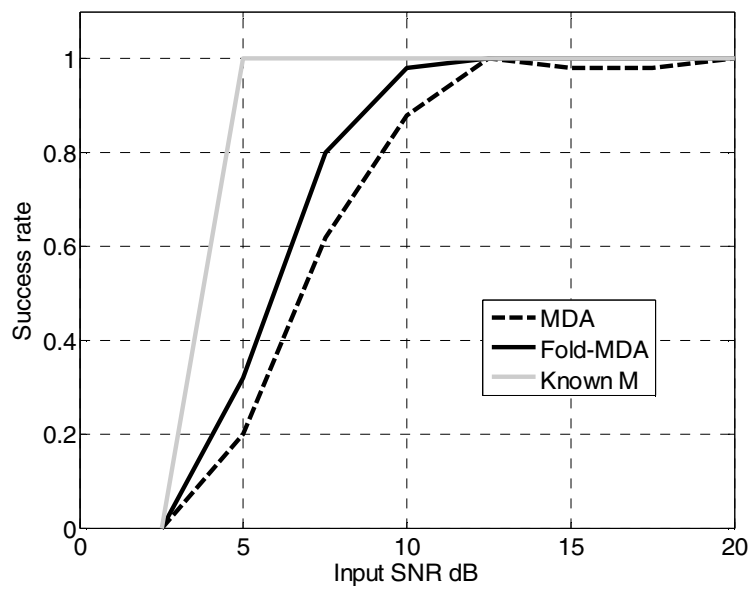

Figure 6. - Success rate as a function of the input SNR.

From figure 6 , we first note that the point at $10 \mathrm{~dB}$ is consistent with the previous plot, the MDA has a $90 \%$ success. We note that the Fold-MDA outperforms MDA: while the MDA fails smoothly, the Fold-MDA supersedes it and reaches a success of $90 \%$ with a gain of more than $1 \mathrm{~dB}$ on the input SNR.

\section{FOLD-MDA APPLIED TO TDR DATA}

In this last section, we present the results of a single case, In this case the data are sampled at $50 \mathrm{MHz}$, using the TDR system [12]. The case of study signal, shown in figure 7, is composed by the superposition of two long Mode $\mathrm{S}$ messages with a relative time delay of ca. $80 \mu \mathrm{s}$. The sources have a quite similar amplitude, (the difference is less than 1 $\mathrm{dB})$.

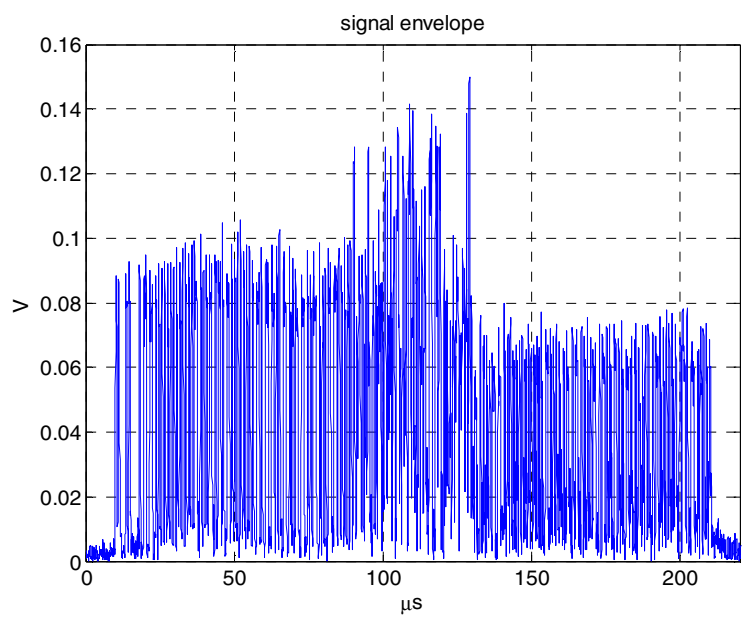

Figure 7. - Signal envelope used as case of study

We note that, due to the large time delay, the MDA failed the separation. The extracted sources still contain both the overlapped signals. Figure 8 shows the first source extracted by MDA. The decoding procedure applied to this signal reveals the reply preamble but the data block still suffer from a substantial overlapping from the second source. Since the two signals mixture has a length of ca. $40 \mu$ s the last 40 bit of the message should be affected by an error decoding output. Figure 9 shows the second source output from MDA. The first source signal amplitude is not negligible and the application of decoding procedure still will finds the first source preamble. The second source preamble cannot be detected, thus it is definitely lost.

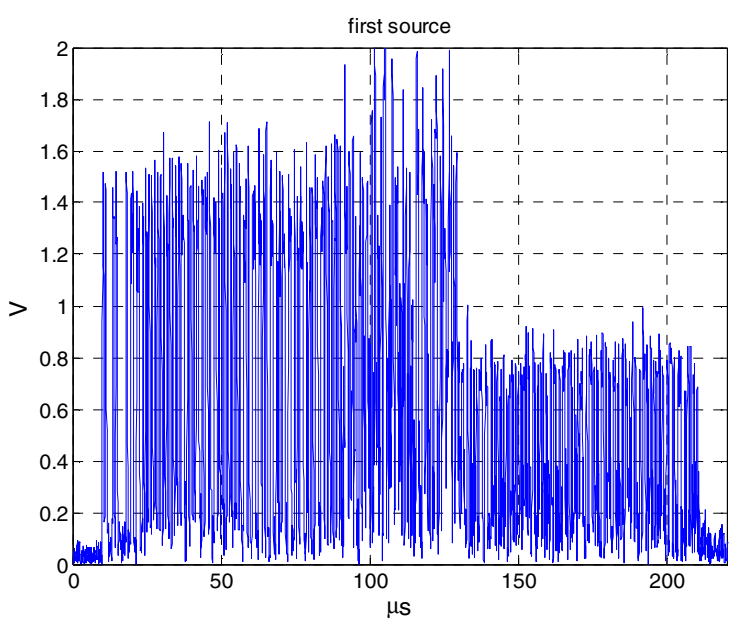

Figure 8. First source from MDA 


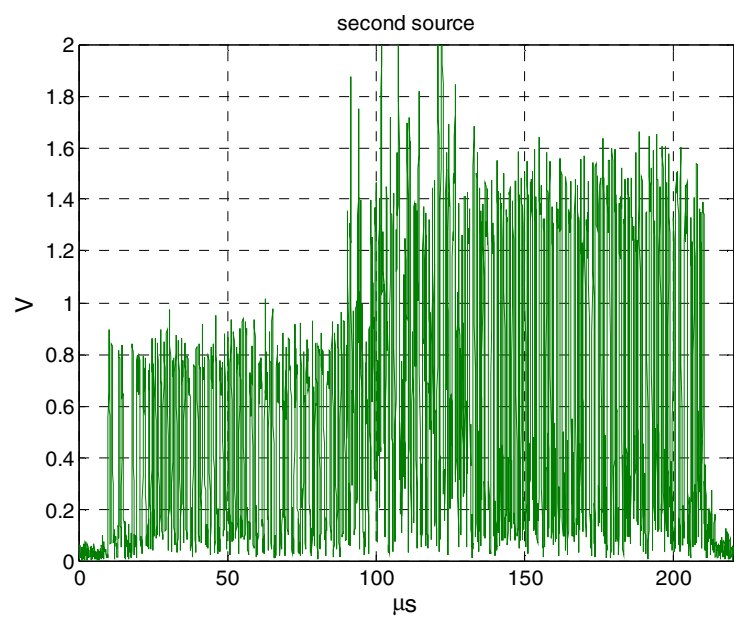

Figure 9. Second source from MDA

Conversely, the Fold-MDA was successful, permitting the sources separation, see figures 10 and 11 . The consequent decoding showed that the first signal is a DF21 message (identity reply to a SSR interrogation) and the second signal is a DF17 (ADS-B message) with a correct CRC.

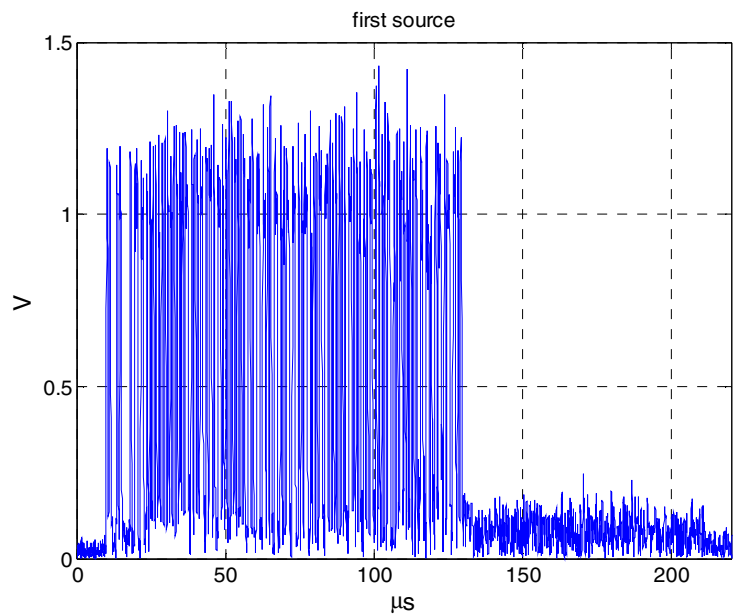

Figure 10. First source from Fold-MDA

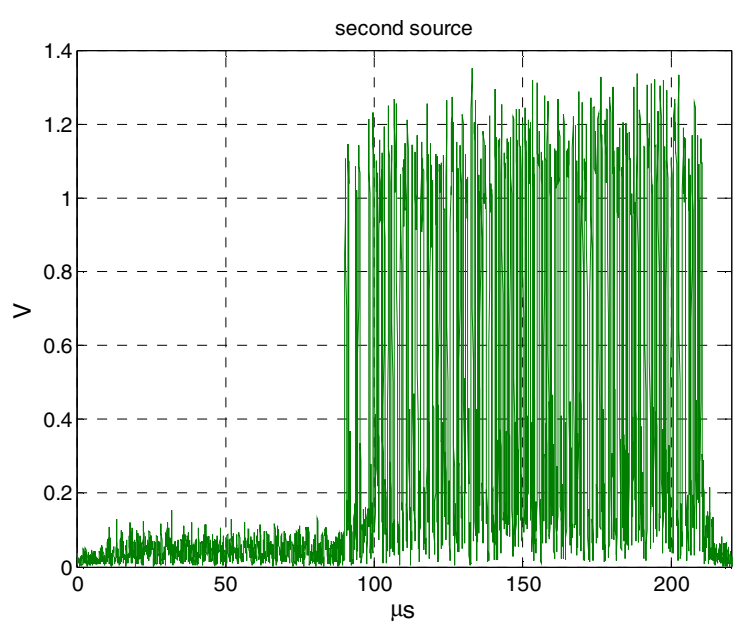

Figure 11. Second source from Fold-MDA

\section{CONCLUSIONS AND FUTURE WORK}

In this paper, We presented the Fold-MDA, an extension of an existing algorithm [1]. This folding pre-processing step extends the robustness of this algorithm and allow us to consider cases not treatable before. In future work we plan to test the algorithm with a larger data base of real overlapping mode S replies in order to perform a full study over all our measured data with comparison to other methods such as [1] and [12].

\section{REFERENCES}

[1] N. Petrochilos, A. van der Veen, "Algebraic algorithms to separate overlapping secondary surveillance radar replies" IEEE Transactions on Signal Processing, Vol. 55, No. 7, July 2007, pp. 3746-3759.

[2] M. Zhou, A. van der Veen, "Improved blind separation algorithm for overlapping secondary surveillance radar replies", in Proceedings of $4^{\text {th }}$ IEEE CAMSAP Int. Workshop, 2011, pp. 181-184.

[3] M. C. Stevens, "Secondary Surveillance Radar" Artech House 1988

[4] P. Bezousek, "A passive radar surveillance system VERA for ATC," in IRS'98, Munich, Germany, 1998.

[5] N. Petrochilos, "Algorithms for Separation of Secondary Surveillance Radar Replies," Ph.D. thesis, University of Nice-Sophia-Antipolis and TU Delft, Nice, France, July 2002 (http://cas.et.tudelft.nl/ nicolas) ISBN 90-407-2371-0.

[6] N. Petrochilos, G. Galati, E.G. Piracci, Separation of SSR signals by array processing in multilateration systems, IEEE Transactions on Aerospace and Electronic Systems, Vol. 45, No. 3 July 2009, pp. 965 982.

[7] N. Petrochilos and P. Comon, "A zero-cumulant random variable and its applications," Signal Processing Magazine, vol. 86, pp. 3334 3338, November 2006.

[8] . Chaumette, P. Comon, D. Muller, "An ICA-Based Technique for Radiating Sources Estimation; Application to Airport Surveillance", IEE Proceedings - Part F, vol. 140, pp. 395-401, December 1993.

[9] A.J. van der Veen and J. Tol, "Separation of zero/constant modulus signals," in Proc. IEEE ICASSP, Munich (FRG), April 1997, pp. 3445--3448.

[10] N. Petrochilos, G. Galati, E.G. Piracci, "Array processing of SSR signals in the multilateration context, a decade survey", TIWDCESAV 2008, 3-5 September 2008, Island of Capri, Vol. I pp. 60-64.

[11] Piracci, E., Galati, G., Pagnini, M.: "ADS-B signals reception: a Software Defined Radio approach", in Proc. Of IEEE MetroAeroSpace, Benevento, Italy, 29-30 May, ISBN 978-1-47992070-9, pp. 543-548.

[12] G. Galati, M. Leonardi, E.G. Piracci, N. Petrochilos, S. Samanta, "The transponder Data Recorder: Implementation and first results", IEEE Aerospace and Electronic Systems magazine, February 2014, volume 29, Number 2

[13] ICAO Annex 10 to the convention on international civil aviation, Vol. IV, 1998

[14] DO260A, RTCA MOPS for 1090 Extended squitter ADS-B and TIS$\mathrm{B}$

[15] N. Petrochilos and P. Comon, "Link between the joint diagonalisation of symmetrical cubes and PARAFAC: an application to secondary surveillance radar," in Proc. of SAM 2006, (Waltham, MA), 12-14 July 2006. 